\title{
APPLICATION OF THE STOCHASTIC MODE-REDUCTION STRATEGY AND A PRIORI PREDICTION OF SYMMETRY BREAKING IN STOCHASTIC SYSTEMS WITH UNDERLYING SYMMETRY*
}

\author{
N. BARLAS ${ }^{\dagger}$ AND I. TIMOFEYEV $\ddagger$ \\ Dedicated to the sixtieth birthday of Professor Andrew Majda
}

\begin{abstract}
We consider application of the stochastic mode-reduction strategy to a particular class of coupled models where a part of self-interactions of the slow variables is given by a rotationally invariant gradient system. The stochastic mode-reduction strategy is utilized to derive stochastic reduced models which yield a simple description of the phenomena resulting from breaking the original rotational symmetry. It is demonstrated that the direction of the symmetry breaking can be predicted a priori without any knowledge of the statistical behavior of the fast modes.
\end{abstract}

Key words. Stochastic modeling, mode reduction, symmetry breaking.

AMS subject classifications. 60H10, 60H35, 65C30.

\section{Introduction}

Recently, stochastic differential equations have been receiving an increasing amount of attention in the mathematical, atmosphere-ocean, and other scientific communities. One important area which emerged from these studies is stochastic modeling and approximation of unresolved degrees of freedom by stochastic dynamics. Straightforward numerical discretizations of complex nonlinear systems often lead to infeasible numerical problems. The vast difference between time scales in the problem is often the main factor which prohibits performing well-resolved numerical simulations. Reduction of the problem's dimensionality, where the non-essential degrees of freedom are represented stochastically, provides one possible computational alternative.

Another area of research in atmosphere-ocean modeling, turbulence, and other areas of nonlinear science is the importance of low-dimensional coherent structures. In particular, the dynamical systems approach has been utilized with some success in lowdimensional truncations of complex models to explain complicated PDE phenomena. In atmosphere-ocean applications, stable low-dimensional structures such as multiple equilibria, periodic orbits, and homoclinic/heteroclinic connections in the phase space of low-dimensional projections have been utilized to explain physical phenomena in observational or numerical data $[4,12,16,20,5,6,28]$. Parametric changes in the behavior of realistic atmosphere-ocean models have also been attributed to bifurcation diagrams of corresponding low-dimensional truncations [25, 29, 3]. Also, effects of random noise are sometimes introduced to model interactions with neglected variables $[7,14,2]$.

The coupled systems analyzed here are designed to address the paradigm of the interaction between coherent structures and noise. In particular, the systems considered here address the effect of coupling and/or perturbations in systems with stable periodic orbits and heteroclinic attractors. A four-dimensional system with stable

\footnotetext{
*Received: December 1, 2008; accepted (in revised version): April 29, 2009.

${ }^{\dagger}$ Department of Mathematics, University of Houston, Houston, TX 77204-3008, (nbarlas@math. uh.edu).

${ }_{\ddagger}$ Department of Mathematics, University of Houston, Houston, TX 77204-3008, (ilya@math. uh.edu). www.math.uh.edu/ ilya
} 
heteroclinic orbits and its random perturbations were considered in detail in [1, 14]. Deterministic systems of this type are not generally chaotic. Instead, under suitable conditions on the eigenvalues of the saddle points, heteroclinic cycles are asymptotically stable and all orbits in their neighborhood spend increasing amount of time near the equilibria and heteroclinic transitions become less frequent. Small random perturbations alter this behavior drastically. Under small random perturbations, the mean exit time from a neighborhood of a saddle point is finite and trajectories undergo heteroclinic transitions with an approximately constant frequency. Coupled systems considered here mimic this situation in a deterministic fashion. In addition, examples presented in this paper elucidate several important properties of a recently developed mode-elimination strategy $[22,23]$ for the high-dimensional dynamical systems with separation of time scales. The stochastic mode-elimination strategy was designed to reduce the dimension of the problem by systematically eliminating fast variables from the equation and replacing them by appropriate stochastic terms. This methodology was successfully applied to small prototype models [24, 21], as well as to larger more realistic atmosphere-ocean systems $[10,9]$.

In this paper we consider low-dimensional models coupled with additional degrees of freedom in an "additive" fashion, i.e., coupling terms can be replaced by damping and additive noise using the stochastic mode-reduction strategy. It is not surprising that coupling with additional degrees of freedom destroys the original symmetry of the truncated low-dimensional system. In this paper, we explore the importance of the underlying deterministic dynamics to demonstrate that the direction of the symmetry breaking can be predicted a priori without any knowledge of the statistical behavior of the fast modes. The "additive" symmetry breaking mechanism described here manifests itself in the statistical description of the slow dynamics. First, we elucidate the symmetry breaking mechanism for gradient systems coupled with additional degrees of freedom. In this case a straightforward analysis yields a simple explanation for the occurrence of the maxima in the joint probability density of the variables of interest. Next, we consider a two-dimensional system with a stable circle of equilibria coupled with two additional variables. Although this system cannot be recast as a gradient system and the signature of the periodic orbit is strong in the coupled model, two peaks occur in the joint density of the slow variables. We demonstrate that the same mechanism is responsible for occurrence of these peaks. In particular, only two points on the stable periodic orbit "survive" the perturbation and these two points can be predicted a priori from the structure of the effective damping.

In the third example we consider a weakly coupled heteroclinic system and its stochastic analog. In particular, we couple the four-dimensional dynamical system with a heteroclinic attractor [1] to the Truncated Burgers-Hopf (TBH) bath. The coupled system mimics the behavior of a randomly perturbed four-dimensional heteroclinic system [14] in a deterministic fashion.

The coupled heteroclinic-TBH model was considered previously in [21] and it was demonstrated that the statistical agreement between the coupled system and the reduced model is nearly perfect for a wide range of parameters. Moreover, it is no surprise that the resulting dynamics has a strong signature of the underlying heteroclinic attractor of the original four-dimensional system. Nevertheless, several phenomena arise as the result of interactions of this four-dimensional system with the TBH bath. In particular, it is demonstrated here that the particular form of coupling can have a profound effect on the statistical description of the coupled dynamics. Statistical features of the coupled model cannot be predicted from the knowledge 
of the heteroclinic dynamics alone, but some of them can be easily explained from the point of view of the stochastic reduced model. Effective stochastic perturbations break the original $O(2)$ symmetry of the heteroclinic system. To explain the symmetry breaking mechanism we utilize the fact that the original four-dimensional system restricted to an invariant plane can be represented as a gradient system. Therefore, the symmetry breaking mechanism is similar to the case of the gradient system and eigenvectors of the effective damping matrix play a crucial role in explaining the statistical distribution of the dynamic variables.

The rest of the paper is organized as follows. In section 2 we utilize a simple gradient system to elucidate the mechanism of symmetry breaking. In section 3 we discuss the symmetry breaking mechanism for the two-dimensional system with a stable periodic orbit. Although this system cannot be recast as a gradient system, it closely resembles the features of the prototype system discussed in section 2 . In section 4 we consider a four-dimensional heteroclinic system coupled with Truncated Burgers-Hopf bath. Detailed derivation of reduced systems is omitted for the brevity of presentation. Details on stochastic mode-reduction can be found in $[22,23]$ and also in [11] and [27].

\section{The gradient system}

To illustrate the symmetry breaking phenomena, we consider a class of stochastic models arising from application of the stochastic mode-reduction strategy to systems where the self-interactions of the slow variables are given by a rotationally-invariant gradient system. Such systems give rise to a stable circle of equilibria in the phase space of the bare projection onto the slow dynamics. In particular, we consider coupled models in which the projection onto the slow variables can be represented as a gradient system

$$
\frac{d}{d t} \mathbf{x}=-\nabla V
$$

with

$$
\mathbf{x}=\left(\begin{array}{c}
x_{1} \\
x_{2}
\end{array}\right), \quad V=V\left(|\mathbf{x}|^{2}\right) .
$$

Here for simplicity we discuss the two-dimensional case, but this approach can be generalized to higher dimensions as well. We consider the potential

$$
V\left(x_{1}, x_{2}\right)=-\frac{1}{2} \mu\left(1-\frac{\alpha_{0}}{2}|\mathbf{x}|^{2}\right)|\mathbf{x}|^{2},
$$

so that the system of equations in (2.1) becomes

$$
\begin{aligned}
& \dot{x}_{1}=\mu\left(1-\alpha_{0}|\mathbf{x}|^{2}\right) x_{1}, \\
& \dot{x}_{2}=\mu\left(1-\alpha_{0}|\mathbf{x}|^{2}\right) x_{2},
\end{aligned}
$$

where $x_{1}^{2}+x_{2}^{2}=\alpha_{0}^{-1}$ is a stable circle of equilibria for this system for $\mu>0$.

We utilize fast variables $u_{1}$ and $u_{2}$ to break the original symmetry of the system

$$
\begin{aligned}
& \dot{x}_{1}=(-\nabla V)_{1}+\lambda A_{1} u_{1} u_{2}, \\
& \dot{x}_{2}=(-\nabla V)_{2}+\lambda B_{1} u_{1} u_{2}, \\
& \dot{u}_{1}=\lambda A_{2} x_{1} u_{2}+\lambda B_{2} x_{2} u_{2}-\gamma_{1} u_{1}+\sigma_{1} \dot{W}_{1}, \\
& \dot{u}_{2}=\lambda A_{3} x_{1} u_{1}+\lambda B_{3} x_{2} u_{1}-\gamma_{2} u_{2}+\sigma_{2} \dot{W}_{2},
\end{aligned}
$$


where $W_{1}, W_{2}$ are independent Wiener processes, $A_{j}$ and $B_{j}, j=1,2,3$ denote known interaction coefficients, and $\lambda$ is introduced explicitly to control the strength of coupling. Here we consider the energy-conserving interactions between the slow variables, $\mathbf{x}$, and the fast variables, $u_{1,2}$. This property can be recast through the interaction coefficients as follows

$$
A_{1}+A_{2}+A_{3}=0, \quad B_{1}+B_{2}+B_{3}=0 .
$$

Under the assumption that the variables $x_{1}, x_{2}$ decorrelate much more slowly than the fast modes, the stochastic mode-reduction strategy can be applied to the system in (2.4) and the reduced model for $\left(x_{1}, x_{2}\right)$ is a stochastic differential equation

$$
d \mathbf{x}=-\nabla V d t-A \mathbf{x} d t+\Sigma d \mathbf{W}
$$

where $\mathbf{W}=\left(W_{1}, W_{2}\right)^{T}$ is a vector of independent Wiener processes, $A$ and $\Sigma$ are $2 \times 2$ damping and diffusion matrices, respectively. To simplify the presentation we consider the case $\gamma_{1}=\gamma_{2}=\gamma$ and $\sigma_{1}=\sigma_{2}=\sigma$, but the argument explaining the symmetry breaking mechanism can be extended when damping and diffusion are not identical. With this simplification the damping and diffusion matrices are given by

$$
\begin{gathered}
A=\frac{\lambda^{2} \sigma^{2}}{4 \gamma^{2}}\left(\begin{array}{cc}
A_{1}^{2} & A_{1} B_{1} \\
A_{1} B_{1} & B_{1}^{2}
\end{array}\right), \\
\Sigma=\frac{\lambda \sigma^{2}}{2 \gamma^{3 / 2}} \frac{1}{\sqrt{A_{1}^{2}+B_{1}^{2}}}\left(\begin{array}{cc}
A_{1}^{2} & A_{1} B_{1} \\
B_{1} A_{1} & B_{1}^{2}
\end{array}\right) .
\end{gathered}
$$

Stochastic mode-reduction strategy $[23,24,21]$ (same as adiabatic elimination of fast variables in [11]) allows for systematic dimension-reduction in systems with separation of time-scales. This approach borrows ideas from earlier works on stochastic differential equations $[18,17,19,19,26,8]$ and utilized asymptotic analysis of the backward (or forward) operator corresponding to the stochastic differential equation in (2.4). In particular, we assume that the Ornstein-Uhlenbeck terms in the equations for $u_{1}$ and $u_{2}$ dominate and consider asymptotic analysis of the backward equation for (2.4) with $\gamma_{i}=\gamma_{i} / \varepsilon, \sigma_{i}=\sigma_{i} / \sqrt{\varepsilon}$. Details of the derivation for various systems are given in $[22,23,21]$.

2.1. Symmetry breaking. It can be shown in general that due to the energy-conserving nature of interactions,

$$
A^{T}=A, \quad \Sigma^{T}=\Sigma,
$$

with the property

$$
\Sigma^{2}=\text { const A. }
$$

Moreover, the damping and diffusion matrices have several additional properties. Firstly, each matrix has only one non-zero eigenvalue. This is the direct consequence of the energy-conserving coupling between the slow and fast variables. It also can be shown that the other eigenvalue of $A$ is positive. Secondly, damping and diffusion matrices have the same eigenvectors, and thus have the same diagonalization 
decomposition which follows from property (2.7). Therefore, matrices $A$ and $\Sigma$ can be diagonalized as follows:

$$
A=R^{-1} \tilde{A} R, \quad \Sigma=R^{-1} \tilde{\Sigma} R, \quad \tilde{\Sigma}^{2}=c \tilde{A},
$$

where $\tilde{A}$ and $\tilde{\Sigma}$ are diagonal matrices of eigenvalues and $R$ is the matrix of eigenvectors. Moreover, $R$ is unitary due to the property (2.6), which implies that the eigenvectors are perpendicular and $R$ can be recast as a rotation matrix, i.e.,

$$
R=\left(\begin{array}{cc}
\cos \psi & \sin \psi \\
-\sin \psi & \cos \psi
\end{array}\right) \text {. }
$$

A particular value of $\psi$ depends only on the choice of interaction coefficients $A_{j}$ and $B_{j}, j=1,2,3$, and can be computed a priori without any knowledge about the behavior of the fast variables. As shown in (2.6), leading order statistics of the fast variables (i.e., $\gamma_{i}$ and $\sigma_{i}, i=1,2$ ) enter as a constant in front of matrices composed of interaction coefficients in the expressions for $A$ and $\Sigma$. Therefore, statistical behavior of the fast variables affects only the magnitude of eigenvalues. We would like to emphasize that properties in (2.7), (2.8), and (2.9) are general; they follow from the properties of the mode-reduction strategy and energy-conserving choice of coupling.

To explain the symmetry breaking we consider a change of variables

$$
\mathbf{y}=R \mathbf{x},
$$

where $R$ is the rotation given in (2.8). This allows the system in (2.5) to be rewritten in a simple form with diagonal damping and forcing

$$
d \mathbf{y}=\mu\left(1-\alpha_{0}|\mathbf{y}|^{2}\right) \mathbf{y} d t-\lambda^{2} \tilde{A} \mathbf{y} d t+\lambda \tilde{\Sigma} d \tilde{\mathbf{W}},
$$

where $\tilde{A}$ and $\tilde{\Sigma}$ are diagonal matrices from (2.8) and $\tilde{\mathbf{W}}=R \mathbf{W}$ is a two-dimensional vector of independent Wiener processes (see [11] for justification). Simultaneous diagonalization of both the damping term and the diffusion term is possible due to the properties of $A$ and $\Sigma$ in (2.8). Furthermore, taking into account the above mentioned fact that one eigenvalue of both $\tilde{A}$ and $\tilde{\Sigma}$ is zero, the system in (2.10) can be rewritten to emphasize the one-dimensional structure of the stochastic perturbation

$$
\begin{aligned}
& d y_{1}=\mu\left(1-\alpha_{0}|\mathbf{y}|^{2}\right) y_{1}, \\
& d y_{2}=\mu\left(1-\alpha_{0}|\mathbf{y}|^{2}\right) y_{2}-\lambda^{2} \tilde{a} y_{2}+\lambda \tilde{\sigma} d \tilde{W}_{2},
\end{aligned}
$$

where $\tilde{a}$ and $\tilde{\sigma}$ are the non-zero eigenvalues of $A$ and $\Sigma$, respectively.

The system in (2.11) can be recast as a stochastic perturbation of a gradient system with a potential which is no longer rotation invariant. The system in (2.5) can also be recast as a stochastic perturbation of a gradient system

$$
d \mathbf{x}=-\nabla U d t+\lambda \Sigma d \mathbf{W},
$$

with

$$
U=-\frac{1}{2} \mu\left(1-\frac{\alpha_{0}}{2}|\mathbf{x}|^{2}\right)|\mathbf{x}|^{2}+\frac{A^{11}}{2} x_{1}^{2}+\frac{A^{22}}{2} x_{2}^{2}+A^{12} x_{1} x_{2},
$$

where $A^{i j}$ are the entries of the damping matrix $A$. The minima of the potential in (2.13) coincide with the intersection of the circle $|\mathbf{x}|^{2}=\alpha_{0}^{-1}$ and the neutral direction of 

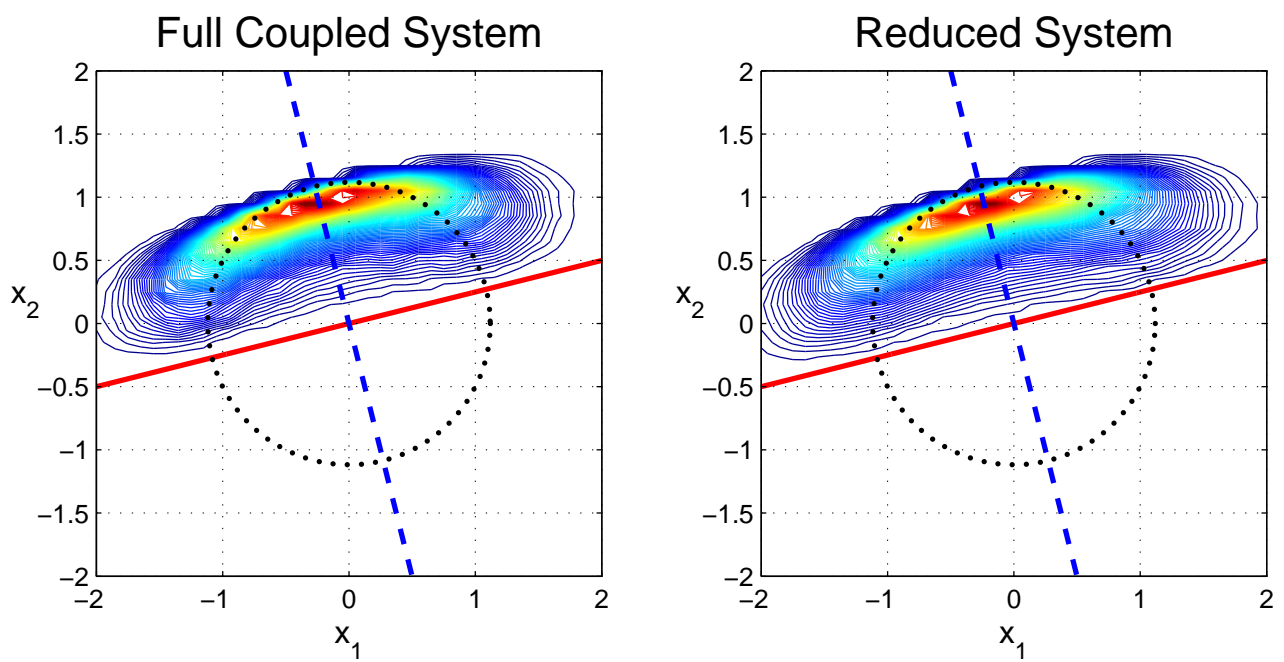

FIG. 2.1. Contour plot of the joint probability density function of $x_{1}, x_{2}$ for the coupled system in (2.4) (left) and the reduced model in (2.5) (right) with parameters in (2.14). The eigenvectors of the matrix $A$ in (2.6) are shown in blue dashed (zero eigenvector) and red solid (non-zero eigenvector) lines. The circle $x_{1}^{2}+x_{2}^{2}=\alpha_{0}^{-1}$ is plotted as dotted line.

the damping matrix, $A$. The direction for the zero eigenvalue is, in turn, perpendicular to the direction of the damping. Therefore, coupling induces a symmetry breaking and a single preferred direction emerges from the interaction between the deterministic dynamics and stochastic terms.

We illustrate symmetry breaking in system (2.4) by plotting the joint probability density function for $x_{1}, x_{2}$ for the coupled and reduced systems. Initial conditions in both simulations were chosen above the eigendirection corresponding to the nonzero eigenvalue. We would like to comment that the reduced system is not ergodic since trajectories cannot cross the line corresponding to the non-zero eigenvector. Therefore, a symmetric part with respect to the direction of the non-zero eigenvalue would also emerge in the joint distribution of $x_{1}, x_{2}$ for generic ensemble simulations of both systems. Parameters in the simulations are

$$
\begin{gathered}
\mu=0.1, \quad \alpha_{0}=0.8, \quad \lambda=0.5, \\
A_{1,2,3}=-2, \quad-2.5,4.5, \quad B_{1,2,3}=-0.5, \quad-0.5,1, \\
\gamma=5, \quad \sigma=3.1622 .
\end{gathered}
$$

The peak in the joint probability density function corresponds to the minimum of the potential in (2.13). Therefore, the position of the peak can be predicted a priori as the point of intersection of the stable circle of equilibria with the eigendirection of the zero eigenvalue.

\section{Low-dimensional system with stable periodic orbit}

Although systems with stable periodic orbits cannot be recast as gradient systems, the argument from section 2 regarding the modification of the potential can be extended to this case as well. To illustrate the symmetry breaking of systems with 
stable periodic orbits, we consider the following system

$$
\begin{aligned}
& \dot{x}_{1}=\mu\left(1-\alpha_{0}|\mathbf{x}|^{2}\right) x_{1}-\left(\alpha+\beta|\mathbf{x}|^{2}\right) x_{2}+\lambda A_{1} u_{1} u_{2}, \\
& \dot{x}_{2}=\mu\left(1-\alpha_{0}|\mathbf{x}|^{2}\right) x_{2}+\left(\alpha+\beta|\mathbf{x}|^{2}\right) x_{1}+\lambda B_{1} u_{1} u_{2}, \\
& \dot{u}_{1}=\lambda A_{2} x_{1} u_{2}+\lambda B_{2} x_{2} u_{2}-\gamma_{1} u_{1}+\sigma_{1} \dot{W}_{1}, \\
& \dot{u}_{2}=\lambda A_{3} x_{1} u_{1}+\lambda B_{3} x_{2} u_{1}-\gamma_{2} u_{2}+\sigma_{2} \dot{W}_{2} .
\end{aligned}
$$

It is easy to show that the system in (3.1) projected onto $x_{1}, x_{2}$,

$$
\begin{aligned}
& \dot{x}_{1}=\mu\left(1-\alpha_{0}|\mathbf{x}|^{2}\right) x_{1}-\left(\alpha+\beta|\mathbf{x}|^{2}\right) x_{2}, \\
& \dot{x}_{2}=\mu\left(1-\alpha_{0}|\mathbf{x}|^{2}\right) x_{2}+\left(\alpha+\beta|\mathbf{x}|^{2}\right) x_{1},
\end{aligned}
$$

possesses a stable periodic orbit

$$
\mathbf{x}(t)=\alpha_{0}^{-1 / 2}(\cos \omega t, \sin \omega t), \quad \text { with } \omega=\alpha+\beta \alpha_{0}^{-1} .
$$

Derivation of the reduced system is similar to the case of the gradient system in section 3 . The reduced system for (3.1) is given by

$$
\begin{aligned}
& \dot{x}_{1}=\mu\left(1-\alpha_{0}|\mathbf{x}|^{2}\right) x_{1}-\left(\alpha+\beta|\mathbf{x}|^{2}\right) x_{2}-A^{11} x_{1}-A^{12} x_{2}+\Sigma_{1} \dot{W}_{1}, \\
& \dot{x}_{2}=\mu\left(1-\alpha_{0}|\mathbf{x}|^{2}\right) x_{2}+\left(\alpha+\beta|\mathbf{x}|^{2}\right) x_{1}-A^{12} x_{1}-A^{22} x_{2}+\Sigma_{2} \dot{W}_{2},
\end{aligned}
$$

where $A^{i j}$ are entries of the damping matrix in (2.6). Therefore, the effective damping is identical to the case of the gradient system and can be diagonalized by a rotation transformation in (2.9). Similar to the example discussed in the previous section, the diffusion matrix can also be diagonalized by the same transformation.

Although the system in (3.2) cannot be recast as a gradient system, parts of the right-hand side of the coupled system and the reduced equations are identical to the gradient system in (2.4) and (2.5), respectively. Therefore, we expect that the peaks in the joint density of $x_{1}, x_{2}$ will occur near the intersection of the circle $x_{1}^{2}+x_{2}^{2}=\alpha_{0}^{-1}$ and the eigenvector corresponding to the zero eigenvalue of the damping matrix. This is confirmed by the numerical simulations with surprising accuracy. The joint probability density for the coupled system and the reduced system is depicted in figure 3.1. Parameters in the simulation were chosen to be similar to the parameters in section 2

$$
\begin{gathered}
\mu=0.1, \quad \alpha_{0}=0.8, \quad \lambda=0.5, \quad \alpha=0.06, \quad \beta=0.05, \\
A_{1,2,3}=-2, \quad-1.5,3.5, \quad B_{1,2,3}=-1.25, \quad-1.2,2.45, \\
\gamma=5, \quad \sigma=3.1622 .
\end{gathered}
$$

The stochastic mode-reduction was designed to reproduce the statistical features of complex models with separation of time-scales. For the coupling strength $\lambda=0.5$ the stochastic mode-reduction strategy is utilized in the correct regime and reproduces statistical features of $x_{1}$ and $x_{2}$ extremely well. Normalized correlation functions of $x_{1,2}$ and $u_{1,2}$ depicted in left part of figure 3.2 demonstrate that the time-scale of $x_{1,2}$ is much slower than for $u_{1,2}$. Statistical agreement of the coupled and reduced models is presented in Table 3.1 and correlation function of $x_{1}$ for two models is depicted in the right part of figure 3.2. Agreement for the correlation function of $x_{2}$ is not presented here only for the brevity of the presentation. Normalized correlation functions were computed as time-averages; correlation function of $f(t)$ is given by 

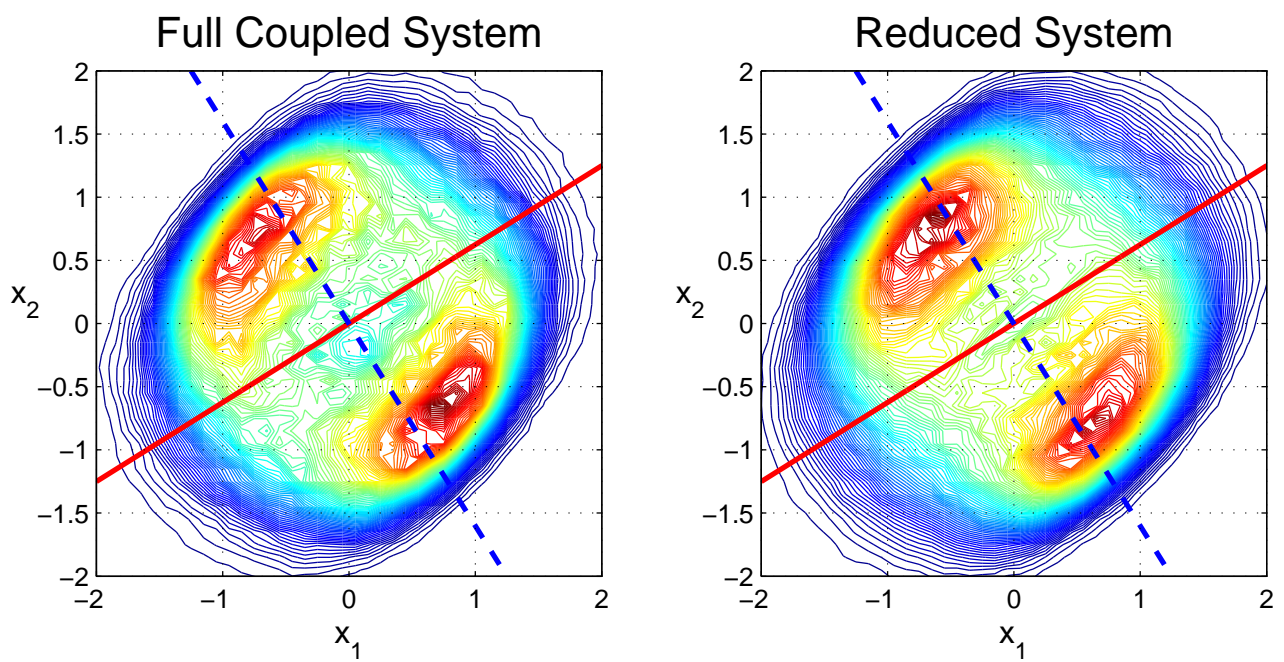

FIG. 3.1. Joint probability density of $x_{1}, x_{2}$ for the coupled system in (3.1) (left) and the reduced model in (3.4) (right) with parameters in (3.5). The eigenvectors of the matrix $A$ in (2.6) are shown in blue dashed (zero eigenvector) and red solid (non-zero eigenvector) lines.

\begin{tabular}{ccccccc}
\hline \hline & $E\left\{x_{1}\right\}$ & $E\left\{x_{2}\right\}$ & $\operatorname{Var}\left\{x_{1}\right\}$ & $\operatorname{Var}\left\{x_{2}\right\}$ & $K\left\{x_{1}\right\}$ & $K\left\{x_{2}\right\}$ \\
\hline Coupled Model & -0.00224 & 0.00339 & 0.57894 & 0.63812 & 2.1703 & 2.0556 \\
Effective Model & -0.00482 & 0.01155 & 0.58613 & 0.65066 & 2.2636 & 2.1143 \\
\hline \hline
\end{tabular}

TABLE 3.1. One-point statistics of $x_{1}$ and $x_{2}$ for the coupled system in (3.1) and reduced system in (3.4) in the regime with $\lambda=0.5 ; K\{y\}=\left\langle(y-\bar{y})^{4}\right\rangle /\left\langle(y-\bar{y})^{2}\right\rangle^{2}$.

$C F(\tau)=\langle(f(t)-\bar{f}))(f(t+\tau)-\bar{f})\rangle_{t}$, where $\bar{f}$ is the mean of $f$ and $\langle\cdot\rangle_{t}$ denotes temporal average. Correlation functions are normalized by the variance so that $C F(0)=1$.

For the coupling strength $\lambda=10$ there is no scale separation between $x_{1,2}$ and $u_{1,2}$ and the stochastic mode-reduction strategy is not supposed to be utilized to explain the statistical behavior of $x_{1}$ and $x_{2}$. In particular, correlation functions of $x_{1,2}$ and $u_{1,2}$ are depicted in the left part of figure 3.3. This figure demonstrates that $x_{1}$ and $x_{2}$ cannot be treated as slow variables since initial decay rates of correlation functions for $x_{1}$ and $u_{1}$ are comparable. This is confirmed by a large discrepancy between the correlation functions of $x_{1}$ and $x_{2}$ in the coupled and reduced models. Only comparison for the correlation function of $x_{1}$ is presented in the right part of figure 3.3 for the brevity of presentation. The correlation function of $x_{2}$ exhibits a similar trend. Therefore, $\lambda=10$ is the regime which is not appropriate for the application of the stochastic mode-reduction strategy. Nevertheless, the reduced model in (3.4) can still be utilized to explain the symmetry breaking phenomena qualitatively. The joint probability density for $x_{1}$ and $x_{2}$ is presented in figure 3.4. Clearly, there is a large discrepancy between the joint distribution of these variables, but the stochastic modereduction predicts the location of the peaks in the joint distribution with surprising accuracy. This example demonstrates that in some cases the predictive power of the 

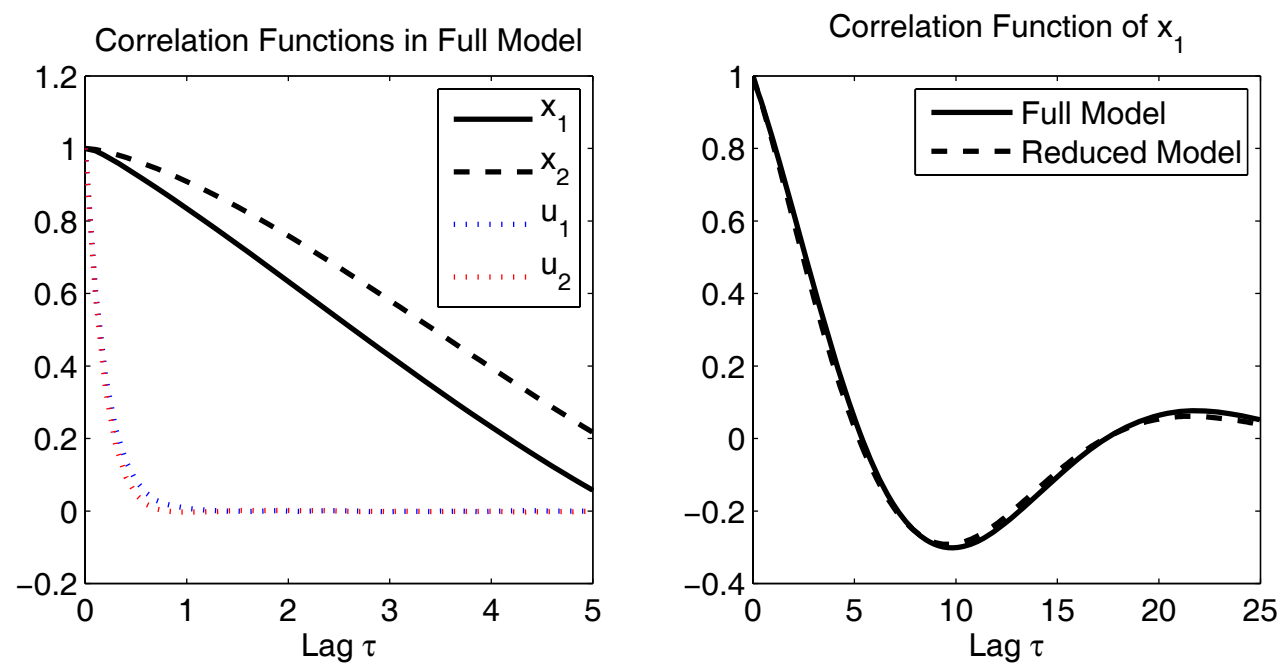

FIG. 3.2. Left: comparison of normalized correlation functions of $x_{1}$ (solid line), $x_{2}$ (dashed line) with correlation functions of $u_{1}, u_{2}$ (dashed line) for simulations of the coupled system in (3.1) with parameters in (3.5); Right: comparison of normalized correlation functions of $x_{1}$ in the simulations of the full coupled system in (3.1) (solid line) in the regime (3.5) and the corresponding
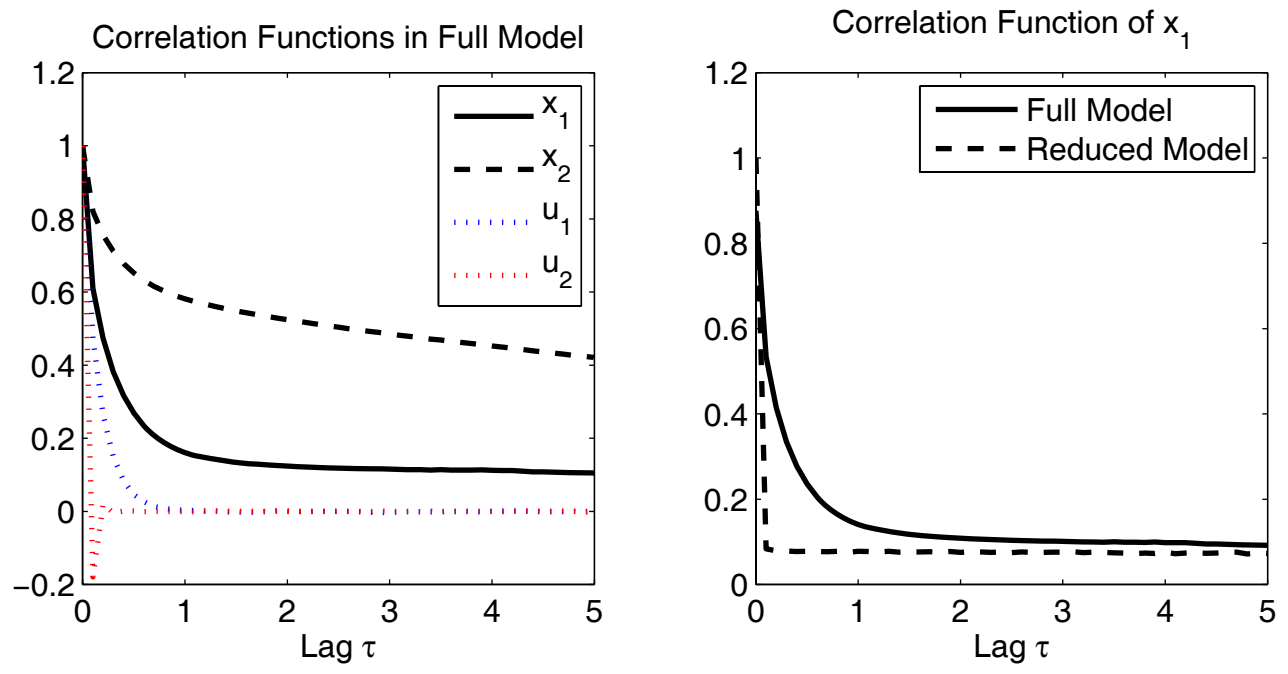

FIG. 3.3. Left: comparison of correlation functions of $x_{1}$ (solid line), $x_{2}$ (dashed line) with correlation functions of $u_{1}, u_{2}$ (dashed line) for simulations of the coupled system in (3.1) with parameters in (3.5), except for the coupling strength $\lambda=10$; Right: comparison of the correlation function of $x_{1}$ in the simulations of the full coupled system in (3.1) (solid line) in the regime (3.5) and the corresponding reduced equation in (3.4) (dashed line).

stochastic mode-reduction extends beyond regimes with scale-separation.

\section{Coupled Heteroclinic system}

Recently in [21] a four-dimensional system of ordinary differential equations coupled to the Truncated Burgers-Hopf heat bath was utilized to investigate the interplay 

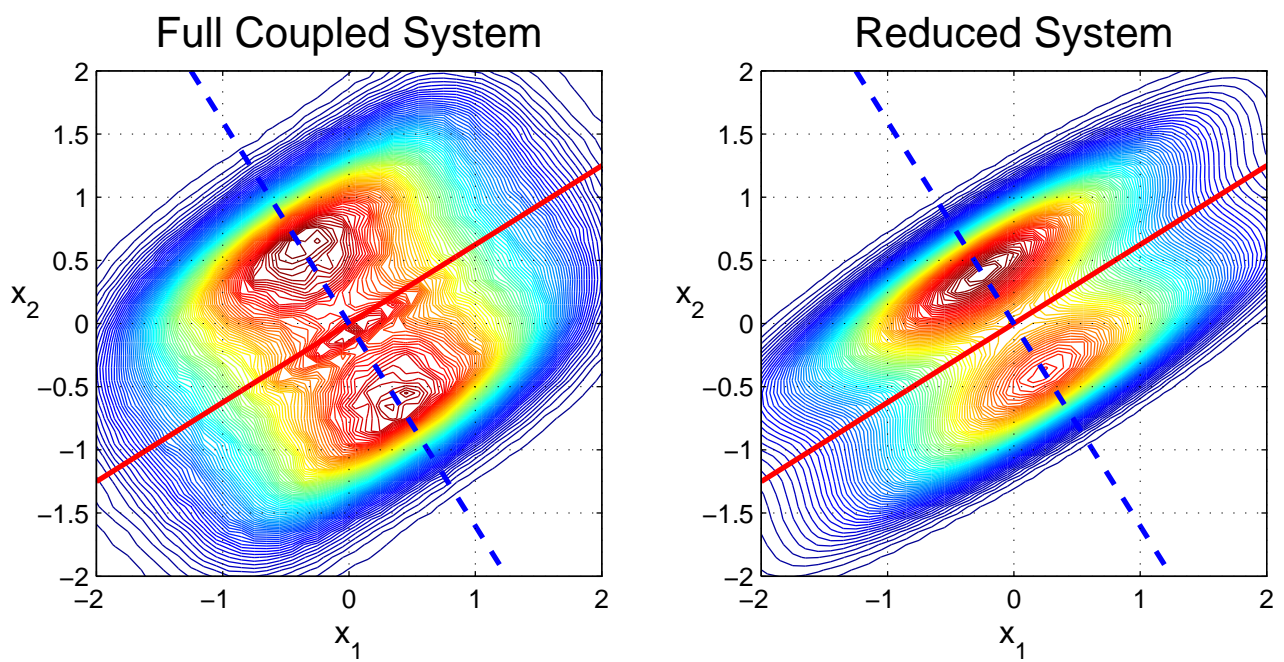

FIG. 3.4. Joint probability density of $x_{1}, x_{2}$ for the coupled system in (3.1) (left) and the reduced model in (3.4) (right) with parameters in (3.5), except for the coupling strength $\lambda=10$. The eigenvectors of the matrix $A$ in (2.6) are shown in blue dashed (zero eigenvector) and red solid (non-zero eigenvector) lines.

between low-dimensional heteroclinic attractor and intrinsic noise. In particular, it was demonstrated that the stochastic mode-reduction performs extremely well in the presence of the underlying heteroclinic attractor in a wide regime of parameters and in various coupling regimes. Here we concentrate on the symmetry breaking mechanism for this model.

The following deterministic four-dimensional system is known to exhibit a wide variety of dynamical properties, including stable heteroclinic cycles, and was utilized as a backbone of the coupled model

$$
\begin{aligned}
& \dot{z_{1}}=z_{1}^{*} z_{2}+\left(\mu_{1}+e_{11}\left|z_{1}\right|^{2}+e_{12}\left|z_{2}\right|^{2}\right) z_{1}, \\
& \dot{z_{2}}=-z_{1}^{2}+\left(\mu_{2}+e_{21}\left|z_{1}\right|^{2}+e_{22}\left|z_{2}\right|^{2}\right) z_{2} .
\end{aligned}
$$

This system was considered in detail in [1]. Stochastic perturbations of this model with emphasis on passage times near equilibria were also considered in $[14,15]$.

Here we give a brief summary of relevant analytical properties of the model in (4.1). One of the general properties of the system in (4.1) is the $O(2)$ symmetry, i.e., the equations are invariant under the reflection $\left(z_{1}, z_{2}\right) \rightarrow\left(z_{1}^{*}, z_{2}^{*}\right)$ and rotation $\left(z_{1}, z_{2}\right) \rightarrow\left(z_{1} e^{i \theta}, z_{2} e^{2 i \theta}\right)$, for any $\theta$. We are mostly interested in the part of the parameter space where there exists a family of stable heteroclinic orbits connecting opposite points on the circle of equilibria $z_{1}=0,\left|z_{2}\right|=\sqrt{-\mu_{2} / e_{22}}$. For detailed discussion of this and other regimes see $[1,13]$. We consider here the following parameter regime

$$
\mu_{1}=0.05, \quad \mu_{2}=0.2, \quad e_{11}=-4, \quad e_{12}=-1, \quad e_{21}=e_{22}=-2 .
$$

The key properties of the system in (4.1) under this choice of parameters are

- The plane $z_{1}=0$ is invariant.

- $z_{1}=0,\left|z_{2}\right|=\sqrt{-\mu_{2} / e_{22}}$ is a circle of equilibria lying in this plane. 
- Every pair of diametrically opposite points on this circle is connected by a heteroclinic cycle.

- Heteroclinic cycles are stable if $\mu_{1}-\mu_{2} e_{12} / e_{22}<0$ and $\mu_{2}>0$, which is satisfied by the above choice of parameters.

The phase of the two points connected by a heteroclinic cycle is a free parameter, which is determined by the initial conditions in each particular realization. model

The system in (4.1) is coupled to the spectrally Truncated Burgers-Hopf (TBH)

$$
\begin{aligned}
& \dot{v}_{k}=-\operatorname{Re} \frac{i k}{2} \sum_{p+q+k=0} \hat{u}_{p}^{*} \hat{u}_{q}^{*}, \quad|p|,|q|,|k| \leq \Lambda, \\
& \dot{w}_{k}=-\operatorname{Im} \frac{i k}{2} \sum_{p+q+k=0} \hat{u}_{p}^{*} \hat{u}_{q}^{*}, \quad|p|,|q|,|k| \leq \Lambda,
\end{aligned}
$$

where $\hat{u}_{k}=v_{k}+i w_{k}$. Coupling is selected to mimic possible energy exchange scenarios in fluid dynamics; the interaction with the bath is constructed in an energy-preserving fashion, but it breaks the original $O(2)$ symmetry of the heteroclinic ODE.

In [21] different types of couplings were considered corresponding to different stochastic perturbations of the model. In the course of those studies various stochastic extensions of the deterministic heteroclinic ODE in (4.1) were derived by the means of the stochastic mode-elimination strategy. Here we consider the additive coupled system

$$
\begin{gathered}
\dot{z}_{1}=z_{1}^{*} z_{2}+\left(\mu_{1}+e_{11}\left|z_{1}\right|^{2}+e_{12}\left|z_{2}\right|^{2}\right) z_{1}+\lambda\left(b^{x_{1} \mid v_{1} w_{1}}+i b^{y_{1} \mid v_{1} w_{1}}\right) v_{1} w_{1}, \\
\dot{z}_{2}=-z_{1}^{2}+\left(\mu_{2}+e_{21}\left|z_{1}\right|^{2}+e_{22}\left|z_{2}\right|^{2}\right) z_{2}+\lambda\left(b^{x_{2} \mid v_{2} w_{2}}+i b^{x_{2} \mid v_{2} w_{2}}\right) v_{2} w_{2}, \\
\dot{u}_{1}=\{\mathrm{TBH}\}+\lambda\left(b^{v_{1} \mid x_{1} w_{1}} x_{1} w_{1}+b^{v_{1} \mid y_{1} w_{1}} y_{1} w_{1}\right)+ \\
\quad i \lambda\left(b^{w_{1} \mid x_{1} v_{1}} x_{1} v_{1}+b^{w_{1} \mid y_{1} v_{1}} y_{1} v_{1}\right), \\
\dot{u}_{2}=\{\mathrm{TBH}\}+\lambda\left(b^{v_{2} \mid x_{2} w_{2}} x_{2} w_{2}+b^{v_{2} \mid y_{2} w_{2}} y_{2} w_{2}\right)+ \\
\quad i \lambda\left(b^{w_{2} \mid x_{2} v_{2}} x_{2} v_{2}+b^{w_{2} \mid y_{2} v_{2}} y_{2} v_{2}\right), \\
\dot{u}_{k}=\{\mathrm{TBH}\}=-\frac{i k}{2} \sum_{p+q+k=0} \hat{u}_{p}^{*} \hat{u}_{q}^{*}, \quad 3 \leq k \leq \Lambda,
\end{gathered}
$$

where $\{\mathrm{TBH}\}$ denotes the Truncated Burgers-Hopf terms introduced in (4.3). The coupling is selected in such a way that the mode $z_{1}$ is coupled only to $u_{1}$ and mode $z_{2}$ is coupled to $u_{2}$; the interaction considered in (4.5) produces corrections of the Ornstein-Uhlenbeck type for the reduced variables. A particular choice of interaction coefficients is presented in Table 4.1 and the parameter $\lambda$ is introduced explicitly to control the strength of interaction between $z_{1}, z_{2}$ and the unresolved modes, $u_{k}$.

The main emphasis of the results presented in [21] was on the performance of the stochastic mode-reduction strategy in the presence of deterministic heteroclinic structures in the low-dimensional truncation. It was demonstrated that, overall, mode-elimination strategy is more robust in the presence of underlying deterministic dynamics. The reduced equation is given by the heteroclinic system in (4.1) plus 


\begin{tabular}{lll}
\hline \hline$b^{x_{1} \mid v_{1} w_{1}}=1$ & $b^{v_{1} \mid x_{1} w_{1}}=-0.75$ & $b^{w_{1} \mid x_{1} v_{1}}=-0.25$ \\
$b^{y_{1} \mid v_{1} w_{1}}=-0.7$ & $b^{v_{1} \mid y_{1} w_{1}}=1$ & $b^{w_{1} \mid y_{1} v_{1}}=-0.3$ \\
$b^{x_{2} \mid v_{2} w_{2}}=1$ & $b^{v_{2} \mid x_{2} w_{2}}=-0.6$ & $b^{w_{2} \mid x_{2} v_{2}}=-0.4$ \\
$b^{y_{2} \mid v_{2} w_{2}}=-0.55$ & $b^{v_{2} \mid y_{2} w_{2}}=1$ & $b^{w_{2} \mid y_{2} v_{2}}=-0.45$ \\
\hline
\end{tabular}

TABLE 4.1. Interaction coefficients for the "additive" model in (4.5). Superscripts are explicitly included to describe particular quadratic interactions between slow and fast variables in the model; the first index is the variable on the left-hand side of the equation in (4.5), the second and third indexes describe the quadratic nonlinearity on the right-hand side of (4.5).

terms of the Ornstein-Uhlenbeck type

$$
\begin{array}{r}
\frac{d}{d t}\left(\begin{array}{c}
x_{1} \\
y_{1}
\end{array}\right)=\left(\begin{array}{c}
x_{1} x_{2}+y_{1} y_{2}+x_{1}\left(\mu_{1}+e_{11} r_{1}^{2}+e_{12} r_{2}^{2}\right) \\
x_{1} y_{2}-y_{1} x_{2}+y_{1}\left(\mu_{1}+e_{11} r_{1}^{2}+e_{12} r_{2}^{2}\right)
\end{array}\right)- \\
\lambda^{2} A_{1}\left(x_{1}, y_{1}\right)^{T}+\lambda \Sigma_{1}\left(\dot{W}_{1}, \dot{W}_{2}\right)^{T}, \\
\frac{d}{d t}\left(\begin{array}{c}
x_{2} \\
y_{2}
\end{array}\right)=\left(\begin{array}{c}
-\left(x_{1}^{2}-y_{1}^{2}\right)+x_{2}\left(\mu_{2}+e_{21} r_{1}^{2}+e_{22} r_{2}^{2}\right) \\
-2 x_{1} y_{1}+y_{2}\left(\mu_{2}+e_{21} r_{1}^{2}+e_{22} r_{2}^{2}\right)
\end{array}\right)- \\
\lambda^{2} A_{2}\left(x_{2}, y_{2}\right)^{T}+\lambda \Sigma_{2}\left(\dot{W}_{3}, \dot{W}_{4}\right)^{T},
\end{array}
$$

where $z_{1}=x_{1}+i y_{1}$ and $z_{2}=x_{2}+i y_{2} . A_{1,2}$ are damping matrices with non-positive eigenvalues, $\Sigma_{1,2}$ are diffusion matrices, and $W_{i}, i=1 . .4$, are independent Wiener processes. Strength of coupling is explicitly emphasized through the parameter $\lambda$. Here we are mostly interested in the relationship between the statistical behavior of the stochastic model in (4.5) and various properties of $A_{1,2}$ and $\Sigma_{1,2}$. In [21] the stochastic extension in (4.5) is systematically derived from the coupled heteroclinic-TBH model by the means of the stochastic mode-elimination strategy. To demonstrate the predictive power of the stochastic mode-elimination strategy we compare statistical behavior of the stochastic equation (4.5) with the behavior of the full coupled heteroclinic-TBH model. Certain statistics of the TBH bath are utilized in deriving the reduced stochastic model. In particular, variance and correlation time of the fast modes are necessary to estimate the bulk statistical influence of the fast modes and approximate the nonlinear interaction by terms of the Ornstein-Uhlenbeck type. The procedure is described in detail in [21] and references therein.

The resulting expressions for damping and diffusion in the stochastic model are

$$
\begin{gathered}
A_{1}=\frac{\lambda^{2} \sigma_{1}^{2}}{4 \gamma_{1}^{2}}\left(\begin{array}{cc}
1 & -0.7 \\
-0.7 & 0.49
\end{array}\right), \quad \Sigma_{1}=\frac{\lambda \sigma_{1}^{2}}{2 \gamma_{1} \sqrt{\gamma_{1}}}\left(\begin{array}{cc}
0.819 & -0.574 \\
-0.574 & 0.401
\end{array}\right), \\
A_{2}=\frac{\lambda^{2} \sigma_{2}^{2}}{4 \gamma_{2}^{2}}\left(\begin{array}{cc}
1 & -0.55 \\
-0.55 & 0.3025
\end{array}\right), \quad \Sigma_{2}=\frac{\lambda \sigma_{2}^{2}}{2 \gamma_{2} \sqrt{\gamma_{2}}}\left(\begin{array}{cc}
0.876 & -0.482 \\
-0.482 & 0.265
\end{array}\right),
\end{gathered}
$$

with the property

$$
\Sigma_{1}^{2}=\frac{\sigma_{1}^{2}}{\gamma_{1}} A_{1}, \quad \Sigma_{2}^{2}=\frac{\sigma_{2}^{2}}{\gamma_{2}} A_{2}
$$


Parameters $\gamma_{1,2}$ and $\sigma_{1,2}$ are related to the statistical properties of the fast modes $u_{1}$, $u_{2}$ and need to be estimated from a single realization of the full coupled system in (4.5).

Parameters $\gamma_{k}$ are determined crudely as the inverse of the area under the graph of the modulus of the correlation function for $u_{k}$ (see $\left.[23,24,21]\right)$. The heat bath, $u_{k}$, achieves an equipartition of energy with mean zero and $\left\langle\operatorname{Re} u_{k}^{2}\right\rangle \approx\left\langle\operatorname{Im} u_{k}^{2}\right\rangle \approx 0.005$ and the parameters $\sigma_{k}$ are determined from the relationship $\sigma_{k}^{2} / \gamma_{k}=\operatorname{Var}\left\{\hat{u}_{k}\right\}$. Estimates for these parameters are

$$
\gamma_{1}=0.4189, \quad \gamma_{2}=0.881, \quad \sigma_{1}=0.0639, \quad \sigma_{2}=0.0925 \text {. }
$$

Statistical behavior of the TBH bath is weakly affected by the coupling for $\lambda \leq 1$, and it was demonstrated in [21] that the above estimates are applicable for a range of coupling strengths. The weak strength of the stochastic terms in (4.5) is misleading, since equation (4.5) represents a dimensional form of the reduced model. Normalization of the dynamic variables by their variances provides an insight into the non-dimensional form of this system. It was demonstrated that for $\lambda \approx 1 \ldots 2$ the stochastic terms are approximately equal to the magnitude of the cubic terms in (4.5). We use $\lambda=0.2$ in the simulations described in this paper.

It was demonstrated in [21] that the reduced stochastic system in (4.5) approximates the statistical behavior of $x_{1}$ and $x_{2}$ in the full coupled system in (4.5) extremely well for a range of coupling strengths, $\lambda$. In contrast with the results presented in [21], here we concentrate on the predictive features of the stochastic mode-elimination approach and explore the connection between the symmetry breaking in reduced models and full systems in more detail.

In particular, we concentrate here on the statistical features of the joint probability density function of $x_{2}, y_{2}$ depicted in figure 4.1. A direction-neutral perturbation of the heteroclinic system in (4.1) would lead to a uniform distribution of the angle inside the circle $x_{2}^{2}+y_{2}^{2}=-\mu_{2} / e_{22}$, since heteroclinic orbits with different angles would be equally represented in the joint probability density function of $x_{2}, y_{2}$. On the other hand, a preferred direction is clearly visible in figure 4.1. This phenomena is better elucidated in the probability density function of the angle $\phi=\operatorname{atan}\left(y_{2} / x_{2}\right)$ depicted in figure 4.2 .

Symmetry breaking in this system can be explained if we consider dynamics in (4.1) and (4.5) restricted to the plane $z_{1}=0$. Recall that this plane is an invariant subspace for the deterministic system in (4.1). For the parameter regime (4.2) under consideration, the system in (4.1) restricted to $z_{1}=0$ possesses a stable circle of equilibria at $x_{2}^{2}+y_{2}^{2}=-\mu_{2} / e_{22}$. Moreover, the vector field is invariant under rotation. The circle of equilibria $z_{1}=0,\left|z_{2}\right|^{2}=-\mu_{2} / e_{22}$ is unstable in the full four-dimensional heteroclinic system with unstable directions perpendicular to the plane $z_{1}=0$.

Following this intuition, we consider the projection of the stochastic system (4.5) onto the plane $z_{1}=0$

$$
d \mathbf{x}=\left(\mu_{2}+e_{22}|\mathbf{x}|^{2}\right) \mathbf{x} d t-\lambda^{2} A_{2} \mathbf{x} d t+\lambda \Sigma_{2} d \mathbf{W}
$$

where $\mathbf{x}=\left(x_{2}, y_{2}\right)$ and $\mathbf{W}$ is a two-dimensional vector of independent Wiener processes. The system in (4.10) is exactly of the same type as the stochastic perturbation of the gradient system considered in section 2. Therefore, coupling induces a symmetry breaking and a single preferred direction emerges from the interaction between the deterministic dynamics and terms derived from the coupling with the TBH bath. This is illustrated in figures 4.1 and 4.2 . 

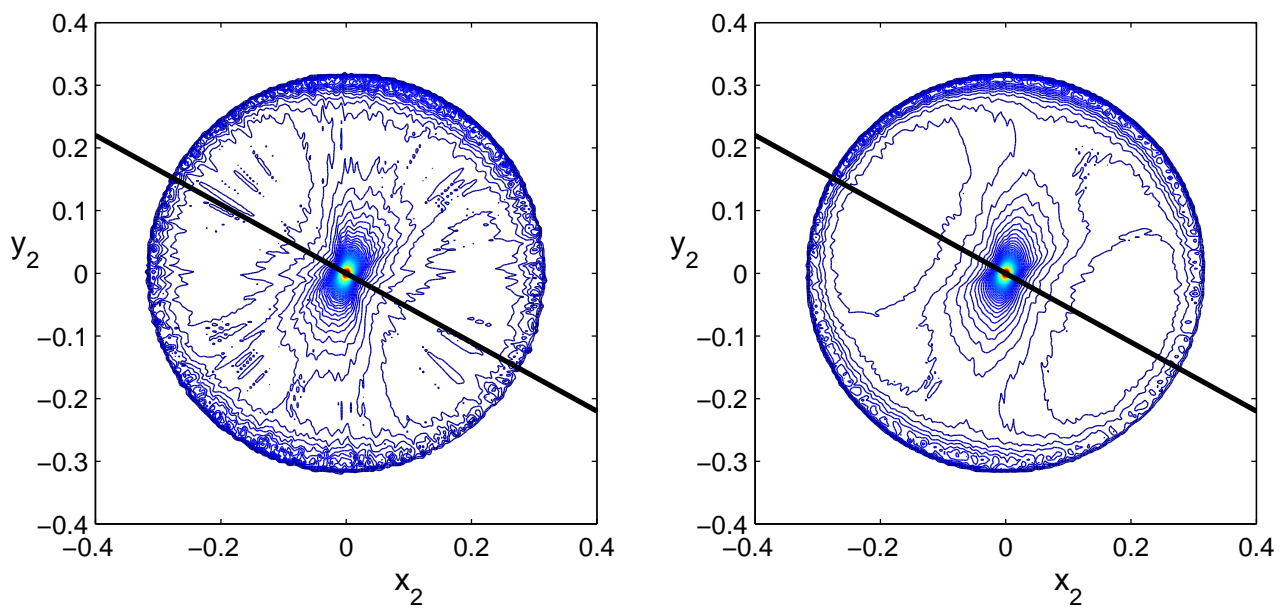

FIG. 4.1. Joint probability density function of $x_{2}, y_{2}$; left column: simulations of the full model in (4.5); right column: simulations of the stochastic system in (4.5); black line: eigenvector corresponding to the non-zero eigenvalue of the damping matrix $A_{2}$.

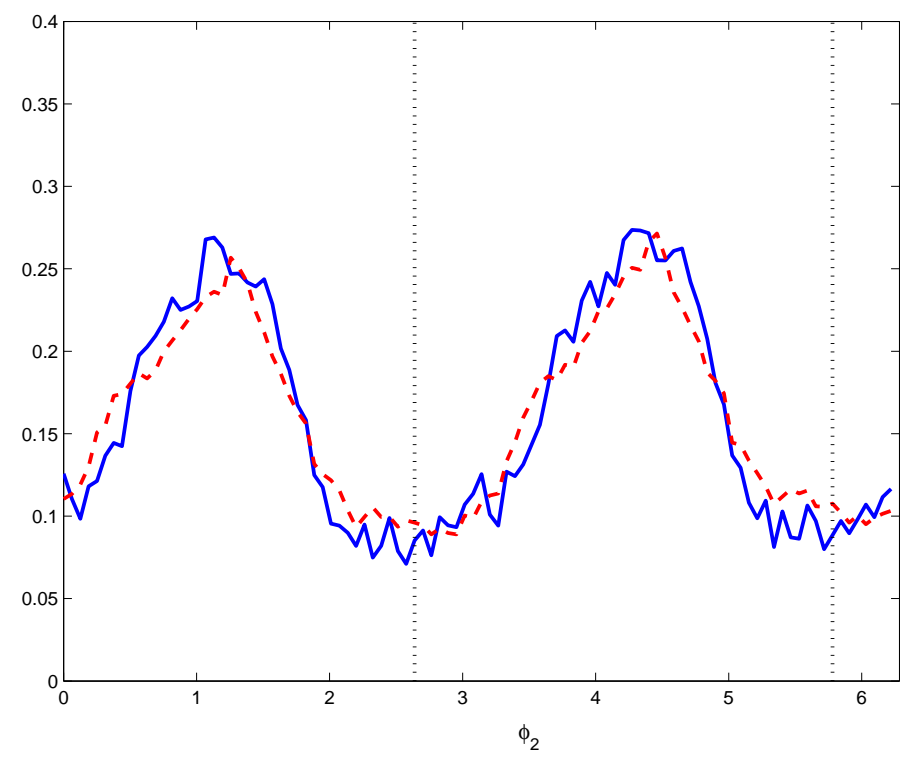

FIG. 4.2. PDF of the phase of $z_{2}$; solid line: full model in (4.5); dashed line: stochastic system in (4.5); vertical dotted line: eigenvector corresponding to the non-zero eigenvalue of the damping matrix $A_{2}$.

\section{Conclusions}

Several coupled systems are presented here as prototype examples elucidating interactions between various deterministic coherent structures and noise. Coupled systems are constructed by coupling a low-dimensional dynamical system with a particular structure with additional variables. It is demonstrated that the coupling breaks 
the original symmetry of the underlying dynamics and a preferred direction emerges. Although not all low-dimensional projections considered here can be recast as gradient systems, $O(2)$ symmetry of the gradient part of the right-hand side plays a crucial role in describing this symmetry breaking mechanism. Furthermore, it is demonstrated that coupling terms can be successfully replaced by stochastic terms utilizing the stochastic mode-reduction technique.

Symmetry breaking can be explained easily in the context of stochastic reduced models since the preferred direction is directly linked to the eigenvectors of the stochastic perturbation. Moreover, these eigenvectors can be computed a priori, without running any computer simulations, thus giving a powerful insight into the statistical behavior of the coupled problem. In addition, it is also demonstrated that the stochastic mode-reduction strategy can predict the preferred direction for systems without scale separation and, thus, far outside regimes of the intended applicability of this technique. Therefore, the stochastic mode-reduction acts as an effective linearization of the full coupled dynamics. We expect that the applicability of the stochastic mode-reduction strategy to problems with symmetry breaking can be extended to more complex systems without scale separation.

Acknowledgments. The authors thank A. J. Majda for valuable comments and suggestions. I. Timofeyev is partially supported by NSF Grants DMS-0713793 and ATM-0417867 and by the DOE Grant DE-FG02-04ER25645.

\section{REFERENCES}

[1] D.J. Armbruster, J. Guckenheimer and P. Holmes, Heteroclinic cycles and modulated traveling waves in systems with O(2) symmetry, Physica D, 29, 257-282, 1988.

[2] G. Branstator and J. Berner, Linear and nonlinear signatures in the planetary wave dynamics of an AGCM: phase space tendencies, J. Atmos. Sci., 62, 1792-1811, 2005.

[3] H. Broer, C. Simo and R. Vitolo, Bifurcations and strange attractores in the Lorenz-84 climate model with seasonal forcing, Nonlinearity, 15, 1205-1267, 2002.

[4] J.G. Charney and J.G. DeVore, Multiple flow equilibria in the atmosphere and blocking, J. Atmos. Sci., 36, 1205-1216, 1979

[5] D.T. Crommelin, Homoclinic dynamics: a scenario for atmospheric ultra-low-frequency variability, J. Atmos. Sci., 59, 1533-1549, 2002.

[6] D.T. Crommelin, Regime transitions and heteroclinic connections in a barotropic atmosphere, J. Atmos. Sci., 60, 229-246, 2003.

[7] H.E. DeSwart and J. Grasman, Effect of stochastic perturbations on a low-order spectral model of the atmospheric circulation, Tellus, 39A, 10-24, 1987.

[8] R.S. Ellis and M.A. Pinsky, The first and second fluid approximations to the linearized Boltzmann equation, J. Math. Pures Appl., 54(9), 125-156, 1975.

[9] C. Franzke and A.J. Majda, Low-order stochastic mode reduction for a prototype atmospheric GCM, J. Atmos. Sci., 63, 457-479, 2006.

[10] C. Franzke, A.J. Majda and E. Vanden-Eijnden, Low-order stochastic mode reduction for a realistic barotropic model climate, J. Atmos. Sci., 62, 1722-1745, 2005.

[11] C.W. Gardiner, Handbook of Stochastic Methods, Springer-Verlag, Berlin, 1985.

[12] M. Ghil and A. Robertson, "Waves" vs. "Particles" in the atmosphere's phase space: a pathway to long-range forecasting? Proc. Nat. Acad. Sci. USA, 99, 2493-2500, 2002.

[13] P. Holmes, J.L. Lumley and G. Berkooz, Turbulence, Coherent Structures, Dynamical Systems, and Symmetry, Cambridge Univ. Press, Cambridge, 1996.

[14] P. Holmes and E. Stone, Noise induced intermittency in a model of a turbulent boundary layer, Physica D, 37, 20-32, 1989.

[15] P. Holmes and E. Stone, Random perturbations of heteroclinic cycles, SIAM J. on Appl. Math., 50(3), 726-743, 1990.

[16] F. Jin and M. Ghil, Intraseasonal oscillations in the extratropics: Hopf bifurcation and topographic instabilities, J. Atmos. Sci., 47, 3007-3022, 1990. 
[17] R.Z. Khasminsky, A limit theorem for the solutions of differential equations with random righthand sides, Theory Prob. Applications, 11, 390-406, 1966.

[18] R.Z. Khasminsky, On stochastic processes defined by differential equations with a small parameter, Theory Prob. Applications, 11, 211-228, 1966.

[19] T.G. Kurtz, A limit theorem for perturbed operator semigroups with applications to random evolution, J. Funct. Anal., 12, 55-67, 1973.

[20] B. Legras and M. Ghil, Persistent anomalies, blocking, and variations in atmospheric predictability, J. Atmos. Sci., 42, 433-471, 1985.

[21] A.J. Majda and I. Timofeyev, Low dimensional chaotic dynamics versus intrinsic stochastic noise: a paradigm model, Physica D, 199, 339-368, 2004.

[22] A.J. Majda, I. Timofeyev and E. Vanden-Eijnden, A mathematics framework for stochastic climate models, Commun. Pure Appl. Math., 54, 891-974, 2001.

[23] A.J. Majda, I. Timofeyev and E. Vanden-Eijnden, A priori tests of a stochastic mode reduction strategy, Physica D, 170, 206-252, 2002.

[24] A.J. Majda, I. Timofeyev and E. Vanden-Eijnden, Systematic strategies for stochastic mode reduction in climate, J. Atmos. Sci., 60(14), 1705-1722, 2003.

[25] B.T. Nadiga and B.P. Luce, Global bifurcation of Shilnikov type in a double-gyre ocean model, J. Phys. Oceanogr., 31, 2669-2690, 2001.

[26] G. Papanicolaou, Some probabilistic problems and methods in singular perturbations, Rocky Mountain J. Math., 6, 653-673, 1976.

[27] G.A. Pavliotis and A.M. Stuart, Multiscale Methods: Averaging and Homogenization, Springer, 2008.

[28] F.M. Selten and G. Branstator, Preferred regime transition routes and evidence for an unstable periodic orbit in a baroclinic model, J. Atmos. Sci., 61, 2267-2282, 2004.

[29] E. Simonnet, M. Ghil and H. Dijkstra, Homoclinic bifurcations in the quasi-geostrophic doublegyre circulation, J. Mar. Res., 63(5), 931-956, 2003. 